\title{
Laboratory assessments of therapeutic platelet inhibition in endovascular neurosurgery: complication prediction using the VerifyNow P2Y12 assay and thromboelastography with platelet mapping
}

\author{
Brian M. Corliss, MD, ${ }^{1}$ Rachel Freedman, BS, ${ }^{2}$ Meghan M. Brennan, MS, MD, ${ }^{3}$ \\ Jessica Smith, MSN, RN, ${ }^{1}$ John D. Nerva, MD, ${ }^{4}$ Neil S. Harris, MD, MBChB, ${ }^{5}$ Adam J. Polifka, MD, ${ }^{1}$ \\ Brian L. Hoh, MD, MBA, ${ }^{1}$ and W. Christopher Fox, MD'1 \\ Departments of ${ }^{1}$ Neurological Surgery, ${ }^{3}$ Anesthesiology, and ${ }^{5}$ Pathology, University of Florida, Gainesville; ${ }^{2}$ University of Florida \\ College of Medicine, Gainesville, Florida; and ${ }^{4}$ Department of Neurological Surgery, Tulane University, Metairie, Louisiana
}

\begin{abstract}
OBJECTIVE Inhibition of platelet aggregation is universally used to prevent thromboembolic complications related to stent placement in endovascular neurosurgery, but excessive inhibition potentiates hemorrhagic complications. Previously, the authors demonstrated that two different commercially available measures of adenosine diphosphate (ADP)dependent platelet inhibition -the VerifyNow P2Y12 clopidogrel assay (measured in platelet reactivity units [PRU]) and maximal amplitude (MA) attributable to ADP activity (MA-ADP) derived from thromboelastography (TEG) with platelet mapping (PM) - yielded wildly different results. This study sought to analyze observed complications to quantify the ideal therapeutic windows for both tests.
\end{abstract}

METHODS Ninety-one patients with simultaneous or near-simultaneous PRU and TEG-PM results who underwent craniocervical endovascular stenting at the authors' institution between September 2015 and November 2017 were identified and retrospectively enrolled. From November 2017 until June 2019, 109 additional patients were prospectively enrolled. For this study, in-hospital thrombotic and hemorrhagic complications (both CNS and non-CNS) were tabulated, and receiver operating characteristic (ROC) curve analysis was used to identify threshold values of the PRU and MAADP for predicting each type of complication.

RESULTS Of the 200 patients enrolled, 7 were excluded because of anemia or thrombocytopenia outside of the test manufacturer's specified ranges and 1 was excluded because they did not have a TEG-PM result. Including complications of all severities, there were a total of $15 \mathrm{CNS}$ thrombotic complications, 1 access-site thrombotic complication, 3 CNS hemorrhages, 8 access-site hemorrhagic complications, and 3 hemorrhagic complications not affecting either the CNS or the access site. ROC curve analysis yielded therapeutic threshold values of 118-144 PRU. The results demonstrated PRU has a significant dose-dependent effect on the rates of thrombosis and hemorrhage. Logistic regression models did not demonstrate statistically significant relationships between the MA-ADP and either thrombosis or hemorrhage. ROC analysis based on these models is of little value and did not identify significant threshold values for MA-ADP.

CONCLUSIONS There continues to be poor correlation between the results of TEG-PM and PRU. PRU accurately predicted complications, with a relatively narrow ideal value range of 118-144. The MA-ADP alone does not appear able to accurately predict either hemorrhagic or thrombotic complications in this group.

https://thejns.org/doi/abs/10.3171/2019.12.JNS192396

KEYWORDS platelet mapping; thromboelastography; clopidogrel; nonresponder; resistance; platelet function test; endovascular neurosurgery; vascular disorders

\footnotetext{
ABBREVIATIONS $A A=$ arachidonic acid; $A D P=$ adenosine diphosphate; $A U C=$ area under the curve; $C B C=$ complete blood count; DAPT = dual antiplatelet therapy; $\mathrm{ICA}=$ internal carotid artery; $\mathrm{MA}=$ maximal amplitude; $\mathrm{PED}=$ Pipeline embolization device; $\mathrm{PFT}$ = platelet function test; $\mathrm{PM}=$ platelet mapping; $\mathrm{PRU}=$ platelet reactivity unit; ROC = receiver operating characteristic; TAPT = triple antiplatelet therapy; TEG = thromboelastography.

SUBMITTED September 1, 2019. ACCEPTED December 2, 2019.
}

INCLUDE WHEN CITING Published online February 21, 2020; DOI: 10.3171/2019.12.JNS192396. 
A S neuroendovascular technology has rapidly expanded, transarterial techniques have replaced open microsurgical techniques in the treatment of many craniocervical vascular pathologies. ${ }^{2,5,20,26,30,31}$ Many endovascular techniques have superior safety and efficacy profiles relative to traditional open methods, but their safety is frequently dependent on therapeutic anticoagulation during the procedure and platelet inhibition during and after the procedure. Typically, these patients require long-term dual antiplatelet therapy (DAPT) in the form of aspirin, an irreversible cyclooxygenase antagonist, and clopidogrel, a prodrug that is metabolized hepatically to an irreversible platelet $\mathrm{P} 2 \mathrm{Y} 12$ adenosine diphosphate (ADP) receptor antagonist.

Metabolism of clopidogrel to its active metabolite is dependent on a number of cytochrome P450 molecules, each of which has multiple polymorphisms identified in humans. Commonly, polymorphisms in a particular cytochrome subtype (CYP2C19) have a profound impact on an individual's ability to successfully metabolize clopidogrel to its active form. As many as 12\%-14\% of American adults are believed to be complete nonresponders to clopidogrel..$^{4,21,25}$ In response to multiple postmarket reports demonstrating increased likelihood of acute and subacute stent thrombosis after coronary angioplasty and stenting ${ }^{13,23}$ in clopidogrel non- or hyporesponders, the US FDA added information on poor metabolizers to clopidogrel drug labels, and subsequently a black-box warning in 2010.

While the concept of testing patients on clopidogrel is well (but not uniformly) accepted among many endovascular neurosurgeons, the data on the use of such tests in neurointervention are limited, and there is no established testing strategy or established algorithm for modifying antiplatelet therapies in patients who demonstrate poor response. Previously, our group demonstrated that significant inconsistency existed between two point-of-care tests of ADP-dependent platelet function, ${ }^{8}$ namely, the VerifyNow P2Y12 assay (measured in platelet reactivity units [PRU]; Accumetrics) and thromboelastography (TEG) with platelet mapping (PM). In the present study, we sought to establish therapeutic ranges of the maximal amplitude (MA) attributable to ADP (MA-ADP), the result from the TEG-PM that reflects ADP-dependent platelet aggregation (and therefore clopidogrel effect), by analyzing complications observed in patients undergoing craniocervical vascular stenting procedures, an analysis previously described by the Thomas Jefferson University group for the PRU in patients undergoing flow diversion for treatment of intracranial aneurysms. ${ }^{9}$

\section{Methods \\ Study Population}

The University of Florida IRB approved the study protocol. All procedures were performed by the senior authors (B.L.H., A.J.P., and W.C.F.). Patients who had undergone cervical carotid artery stenting, intracranial stenting with or without aneurysm coiling, flow diversion with or without aneurysm coiling, or venous sinus stenting while on DAPT and who had preoperative PRU and TEG-PM results obtained simultaneously (or nearly simultaneously) were enrolled retrospectively under a waiver of informed consent. The retrospective enrollment start date was chosen to be September 2015, as this was when TEG-PM technology was introduced to our department. After the protocol approval date in November 2017, patients or their representatives individually consented and patients were enrolled prospectively after they had provided informed consent for one of the procedures above. Patient enrollment was completed in June 2019.

DAPT regimens were implemented at the discretion of the attending surgeon. Typically, elective procedures were performed approximately 7 days after initiation of DAPT, consisting of 81 or $325 \mathrm{mg}$ of aspirin daily plus $75 \mathrm{mg}$ of clopidogrel daily. For urgent or emergent procedures, patients were loaded with aspirin and clopidogrel, usually at least $650 \mathrm{mg}$ and $300 \mathrm{mg}$, respectively, with or without augmentation with intravenous platelet antagonists (e.g., abciximab). Transitioning from clopidogrel to a different ADP receptor antagonist (such as prasugrel or ticagrelor) was conducted at the discretion of the attending surgeon. There was no protocol based on either the PRU or TEGPM for guiding transition from one agent to another.

\section{Data Collection and Analysis}

Once patients were enrolled, simultaneously collected PRU and TEG-PM results were tabulated. The most recent complete blood count (CBC) result obtained prior to the PRU result was also recorded, and patients were excluded from the analysis if the hematocrit level was < $30 \%$ or platelet count $<115,000 / \mathrm{mm}^{3}$, as these values are outside of the validated reference range from the PRU test manufacturer. The PRU is known to be inaccurate below these values. ${ }^{15}$ If no preoperative $\mathrm{CBC}$ was available, the immediate postprocedure $\mathrm{CBC}$ was referenced.

In addition to visualized curves depicting clot formation graphically, the TEG-PM results contain multiple parameters relating to enzymatic coagulation, baseline platelet function, arachidonic acid (AA) pathway-mediated platelet function (i.e., platelet inhibition related to aspirin), and ADP receptor pathway-mediated platelet function (i.e., platelet inhibition related to clopidogrel). For the current analysis, the MA-ADP was the primary subcomponent of the TEG-PM utilized.

In-hospital complications for retrospectively enrolled patients were assessed by review of operative reports, hospital progress notes, radiology reports, and discharge summaries. These sources were reviewed for prospectively enrolled patients as well, and were supplemented with physical examinations and review of pertinent imaging findings (performed by B.M.C. and R.F.). Complications were dichotomized (present or absent) and categorized as either hemorrhagic or thrombotic. Hemorrhagic complications consisted of CNS hemorrhage, access-site hemorrhage, or other hemorrhage (e.g., gastrointestinal hemorrhage or epistaxis). Thrombotic complications consisted of either CNS thromboses or access vessel thrombosis. Thromboses believed to be unrelated to the procedure (e.g., deep vein thromboses) were not considered. CNS or systemic complications of all severity levels were included. Access-site complications were included if they 
TABLE 1. Aneurysm patient and treatment characteristics

\begin{tabular}{|c|c|}
\hline Variable & No. $(\%)$ \\
\hline No. of patients w/ aneurysms & 124 \\
\hline Mean age $\pm S D$, yrs & $59.6 \pm 14$ \\
\hline Mean BMI $\pm \mathrm{SD}, \mathrm{kg} / \mathrm{m}^{2}$ & $28.7 \pm 6.1$ \\
\hline Females & $98(79.0)$ \\
\hline \multicolumn{2}{|l|}{ Aneurysm presentation } \\
\hline Recurrent/residual & $35(28.2)$ \\
\hline Headache/migraine & $31(25.0)$ \\
\hline Incidental & $31(25.0)$ \\
\hline Ischemia/TIA & $8(6.5)$ \\
\hline $\mathrm{SAH}$ & $7(5.6)$ \\
\hline Cranial neuropathy & $6(4.8)$ \\
\hline Dizziness & $4(3.2)$ \\
\hline Screening & $1(0.8)$ \\
\hline Seizure & $1(0.8)$ \\
\hline \multicolumn{2}{|l|}{ Access site* } \\
\hline Femoral & $124(100.0)$ \\
\hline Radial & $1(0.8)$ \\
\hline Brachial & $1(0.8)$ \\
\hline \multicolumn{2}{|l|}{ Sheath size, $\mathrm{Fr}$} \\
\hline 8 & $88(71.0)$ \\
\hline 6 & $35(28.2)$ \\
\hline Other/multiple & $1(0.8)$ \\
\hline \multicolumn{2}{|l|}{ Closure device } \\
\hline Angio-Seal & $110(88.7)$ \\
\hline Mynx & $8(6.5)$ \\
\hline Manual pressure & $3(2.4)$ \\
\hline Multiple devices & $2(1.6)$ \\
\hline Other & $1(0.8)$ \\
\hline
\end{tabular}

$\mathrm{SAH}=$ subarachnoid hemorrhage; $\mathrm{TIA}=$ transient ischemic attack.

* Two patients had multiple access sites, i.e., femoral and radial for one and femoral and brachial for the other.

resulted in blood product transfusion, vascular surgical consultation with or without intervention at the access site, prolonged hospital length of stay, or a change in prescribed follow-up (for example, if follow-up vascular ultrasonography was recommended, a nonroutine part of our follow-up practice).

\section{Statistical Analyses}

Histogram analyses of MA-ADP and PRU values demonstrated them to be normally distributed; therefore, statistical analyses comparing sample means were performed using the Student t-test. Linear regression analysis was used to identify correlation between the PRU and MA-ADP. Receiver operating characteristic (ROC) curve analysis was used to determine therapeutic cutoff values of PRU and MA-ADP for both hemorrhagic and thrombotic complications. Pre-IRB approval power calculation identified a necessary sample size of 160 subjects for this analysis, assuming an $\alpha$ of $0.05, \beta$ of 0.20 , overall complication rate of $10 \%$, and area under the curve (AUC)
TABLE 2. Carotid stenting patient and treatment characteristics ( $n=41)$

\begin{tabular}{|c|c|}
\hline Variable & No. $(\%)$ \\
\hline Mean age $\pm S D$, yrs & $66.4 \pm 11$ \\
\hline Mean BMI $\pm \mathrm{SD}, \mathrm{kg} / \mathrm{m}^{2}$ & $29.4 \pm 6.2$ \\
\hline Males & $27(65.8)$ \\
\hline \multicolumn{2}{|l|}{ Presentation } \\
\hline Symptomatic & $38(92.7)$ \\
\hline Asymptomatic & $3(7.3)$ \\
\hline \multicolumn{2}{|l|}{ Degree of stenosis* } \\
\hline$<50 \%$ & $0(0.0)$ \\
\hline $50 \%-69 \%$ & $2(4.9)$ \\
\hline $70 \%-89 \%$ & $25(61)$ \\
\hline$\geq 90 \%$ & $14(34.1)$ \\
\hline \multicolumn{2}{|l|}{ Distal protection device } \\
\hline Yes & 39 (95.1) \\
\hline No & $2(4.9)$ \\
\hline \multicolumn{2}{|l|}{ Access site } \\
\hline Femoral & 40 (97.6) \\
\hline Radial & $1(2.4)$ \\
\hline Brachial & $0(0.0)$ \\
\hline \multicolumn{2}{|l|}{ Sheath size, Fr } \\
\hline 8 & $38(92.7)$ \\
\hline 6 & $3(7.3)$ \\
\hline Other/multiple & $0(0.0)$ \\
\hline \multicolumn{2}{|l|}{ Closure device } \\
\hline Angio-Seal & $37(90.2)$ \\
\hline Mynx & $0(0.0)$ \\
\hline Manual pressure & $3(7.3)$ \\
\hline TR Band & $1(2.4)$ \\
\hline Other & $0(0.0)$ \\
\hline
\end{tabular}

* Degree of carotid stenosis was determined angiographically according to North American Symptomatic Carotid Endarterectomy Trial criteria.

of 0.75 . We planned to enroll 200 patients to account for patient withdrawal or exclusion due to thrombocytopenia, anemia, or change in procedure performed (e.g., no stent placed during aneurysm coil embolization). Patients were excluded from the complication analysis if they did not receive a stent.

\section{Results}

Ninety-one patients were retrospectively enrolled and 109 prospectively enrolled for a total enrollment of 200 patients. Of these, 8 results were excluded from analysis due to thrombocytopenia, anemia, or missing data from either the PRU or TEG-PM. One hundred seventy-two patients ultimately underwent placement of a stent. Patient demographic information is outlined in Tables 1 and 2. Details on treated aneurysms and procedure specifics are outlined in Tables 3 and 4, respectively. There were 15 CNS thrombotic complications and 1 femoral artery thrombosis requiring vascular intervention (Supplemen- 
TABLE 3. Treated aneurysm locations and size $(n=136)$

\begin{tabular}{ccc}
\hline Aneurysm Location & No. $(\%)$ & Mean Size \pm SD $(\mathrm{mm})^{*}$ \\
\hline Anterior circulation & $109(80.1)$ & $7.8 \pm 4.7$ \\
\hline Cavernous & $8(5.9)$ & $14.2 \pm 3.7$ \\
\hline OphA $\dagger$ & $51(37.5)$ & $7.3 \pm 4.7$ \\
\hline PCoM & $20(14.7)$ & $6.4 \pm 2.7$ \\
\hline Other supraclinoid ICA $\ddagger$ & $11(8.1)$ & $7.1 \pm 3.0$ \\
\hline ACoM & $11(8.1)$ & $5.7 \pm 2.5$ \\
\hline Other ACA/MCA & $8(5.9)$ & $11.6 \pm 6.2$ \\
\hline Posterior circulation & $27(19.9)$ & $8.8 \pm 6.5$ \\
\hline PICA & $2(1.5)$ & $9.6 \pm 10$ \\
\hline VBA§ & $11(8.1)$ & $9.0 \pm 7.4$ \\
\hline SCA & $3(2.2)$ & $6.5 \pm 1.4$ \\
\hline BTA & $12(8.8)$ & $9.0 \pm 6.5$ \\
\hline PCA/other & $0(0.0)$ & NA \\
\hline
\end{tabular}

$\mathrm{ACA}=$ anterior cerebral artery; $\mathrm{ACoM}=$ anterior communicating artery; $\mathrm{BTA}=$ basilar terminus aneurysm; $M C A=$ middle cerebral artery; $N A=$ not applicable; $\mathrm{OphA}=$ ophthalmic artery; $\mathrm{PCA}=$ posterior cerebral artery; $\mathrm{PCoM}=$ posterior communicating artery; PICA = posterior inferior cerebellar artery; SCA = superior cerebellar artery; VBA = vertebrobasilar artery.

${ }^{*}$ Aneurysm size includes only largest treated aneurysm in patients undergoing embolization of multiple aneurysms.

† Ophthalmic segment aneurysms included ophthalmic, superior hypophyseal, paraophthalmic, and other aneurysms.

$\ddagger$ Other supraclinoid ICA aneurysms included anterior choroidal, ICA terminus, fusiform ICA, or other non-PCoM communicating-segment ICA aneurysms. $\S$ VBA aneurysms included non-PICA vertebral artery aneurysms, vertebrobasilar junction aneurysms, and basilar trunk aneurysms.

tal Tables 1 and 3). There were 3 CNS hemorrhages, 8 access-site hemorrhages, and 3 non-CNS/nonaccess-site hemorrhages (1 episode of epistaxis, 2 episodes of gastrointestinal hemorrhage; Supplemental Tables 2 and 3). One patient had both CNS thrombosis and CNS hemorrhage, and one had both CNS thrombosis and groin site hemorrhage.

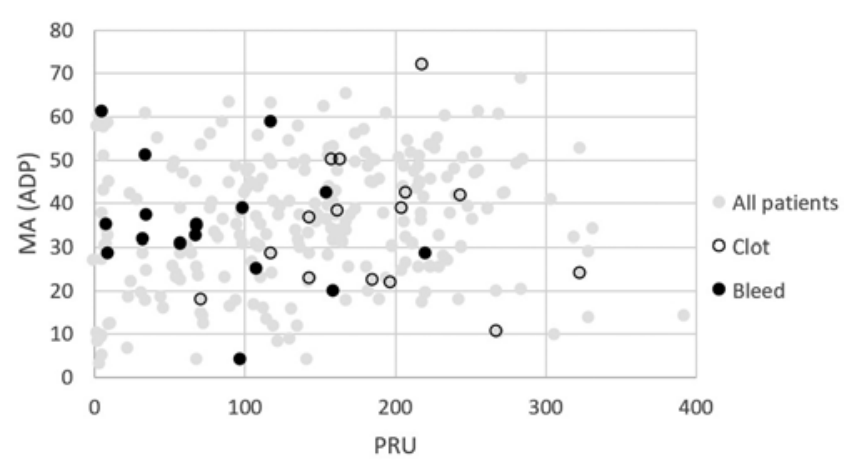

FIG. 1. Scatterplot demonstrating paired MA-ADP and PRU results for all patients, including multiple results from the same patient (246 pairs of results in 192 patients). There is no significant linear correlation between MA-ADP and PRU values.

Two hundred forty-six pairs of results were obtained in the 192 patients included; these results are plotted graphically in Fig. 1. There was no significant correlation between the PRU and the MA-ADP, which was consistent with the results of our previous publication. ${ }^{8}$ This remained true when multiple results from the same patient were excluded and only the final predischarge pair was used. Final paired test results from the 172 patients who ultimately underwent stent placement are shown in Fig. 2; again, there was no statistical correlation.

For patients who underwent placement of a stent, the mean MA-ADP in those who suffered thrombotic complications was statistically no different than the sample mean $(34.5 \pm 15.2 \mathrm{~mm}$ vs $35.1 \pm 13.5 \mathrm{~mm}, \mathrm{p}=0.87)$, while PRU values were significantly higher in those patients who suffered thrombotic complications (180 \pm 67 vs $142 \pm 77$, $\mathrm{p}=$ 0.048). In that same subset of patients, the mean MA-ADP in those who suffered hemorrhagic complications was, again, statistically no different than the sample mean (35.7 \pm 14.4 vs $35.1 \pm 13.5 \mathrm{~mm}, \mathrm{p}=0.88$ ), while the PRU was significantly lower $(71 \pm 53$ vs $142 \pm 77, p=0.0002)$. Furthermore, the PRU showed a dose-dependent effect on the

TABLE 4. Treatment methods for anterior and posterior circulation aneurysms

\begin{tabular}{lrrrcc}
\hline Aneurysm Location & PED & PED + Coil & Stent + Coil & Stent w/o Coil & PulseRider + Coil \\
\hline Anterior circulation & & & & & \\
\hline Cavernous ICA & 6 & 2 & 0 & 0 & 0 \\
\hline OphA & 39 & 12 & 0 & 0 & 0 \\
\hline PCoM & 15 & 4 & 1 & 0 & 0 \\
\hline Other ICA & 4 & 3 & 4 & 0 & 0 \\
\hline ACoM & 0 & 0 & 11 & 0 & 0 \\
\hline Other ACA/MCA & 6 & 0 & 2 & 0 & 0 \\
\hline Posterior circulation & & & & & 0 \\
\hline PICA & 0 & 1 & 0 & 1 & 0 \\
\hline VBA & 6 & 2 & 2 & 0 & 0 \\
\hline SCA & 0 & 0 & 3 & 0 & \\
\hline BTA & 0 & 0 & 9 & 0 & 0 \\
\hline PCA/other & 0 & 0 & 0 & 0 & \\
\hline
\end{tabular}




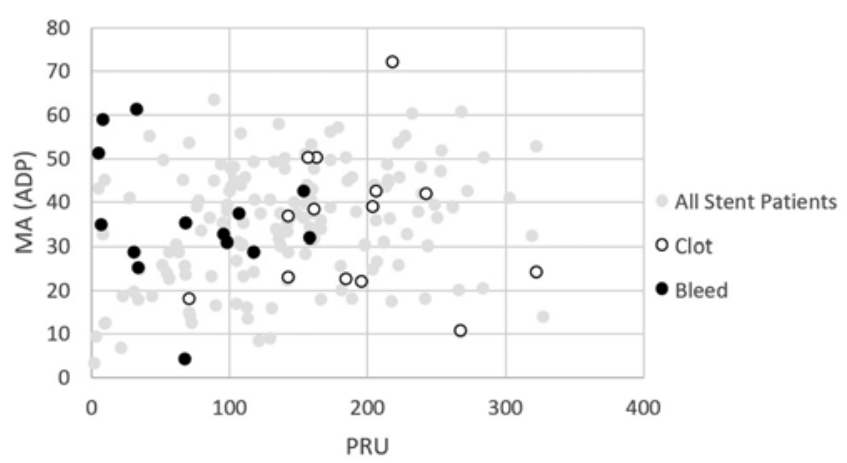

FIG. 2. Scatterplot demonstrating paired MA-ADP and PRU results for patients undergoing stent placement. One pair of results was plotted for each patient. In patients with multiple results, only the final result is plotted, i.e., the result on which the final clinical decision regarding antiplatelet therapies was made.

rate of both thrombosis and hemorrhage (Fig. 3), findings that were not observed for the MA-ADP.

Finally, the ROC analysis identified threshold values of 118 and 144 PRU to avoid hemorrhagic (sensitivity 86\%, specificity $61 \%$, AUC 0.79 ; Fig. 4A) and thrombotic (sensitivity $81 \%$, specificity $56 \%$, AUC 0.66 ; Fig. 4B) complications, respectively, suggesting an ideal therapeutic window consistent with that published previously by Daou and colleagues. ${ }^{9}$ The same analysis did not identify significant threshold values for MA-ADP due to poor fits of the logistical regression model and very low areas under the ROC curves. The best model yielded threshold values of $<23.9$ $\mathrm{mm}$ to prevent thromboses and $>24.8 \mathrm{~mm}$ to prevent hemorrhages, an obviously invalid result as the "high" threshold value is lower than the "low" threshold value. A less specific (and still not statistically significant) range from the same ROC curve would be threshold values of 24.8 $\mathrm{mm}$ to avoid hemorrhages (sensitivity 93\%, specificity 26\%, AUC 0.49; Fig. 4C) and $42.3 \mathrm{~mm}$ to avoid thromboses (sensitivity $81 \%$, specificity $34 \%$, AUC 0.54 ; Fig. 4D).

\section{Discussion}

In-stent thrombosis is a potentially devastating complication following endovascular neurosurgical procedures. It follows from studies in the coronary stenting literature that such complications may be reduced by $75 \%-80 \%$ with the use of DAPT in these patients, ${ }^{7}$ but the high number of clopidogrel nonresponders and hyporesponders in the American population make it difficult for surgeons to know that any given patient's platelets will be sufficiently inhibited to safely perform such a procedure. The FDA recognized this shortcoming in 2010 when it applied a boxed warning to clopidogrel packaging, which suggests practitioners utilize genome-based testing aimed at identifying patients with specific CYP2C19 polymorphisms (this warning was not aimed at the neurointervention setting in particular). Unfortunately, genomic testing has several shortcomings. First, clopidogrel hyporesponsiveness is not solely determined by CYP2C19 activity. Myriad polymorphisms in a number of genes involved in clopidogrel absorption, metabolism, and subsequent platelet response, all of varying prevalences in various ethnic and racial populations, have been implicated in this phenomenon. ${ }^{7}$ These genes are not tested for by the most widely available genome-based tests, which are specific for CYP2C19 polymorphisms. Second, genomic tests are time consuming and may not be readily available at some institutions, which makes this sort of testing of limited utility for patients undergoing urgent procedures. And

\section{A Hemorrhage rate as a function of PRU}

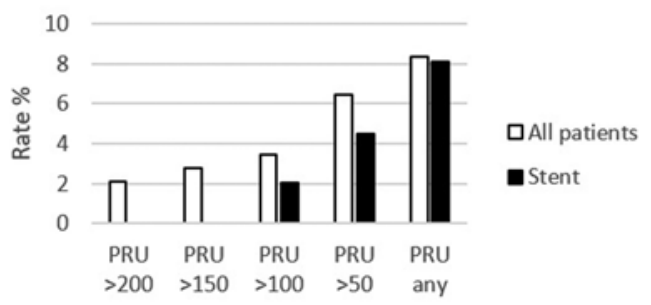

C Hemorrhage rate (\%) as a function of MA (ADP)

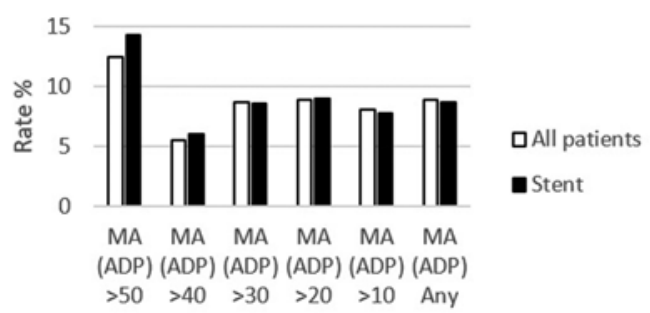

\section{B Thrombosis rate as a function of} PRU

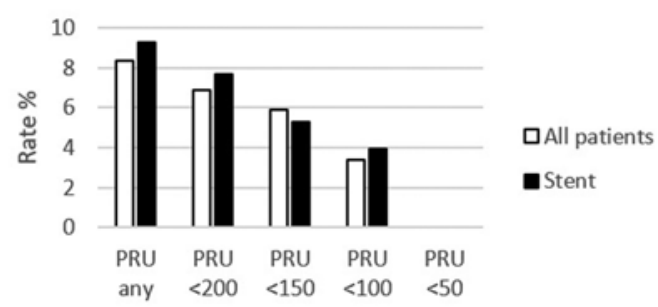

\section{$D^{\text {Thrombosis rate (\%) as a function of }}$ MA (ADP)}

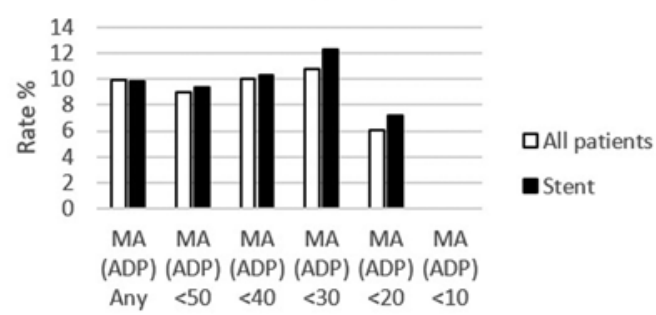

FIG. 3. Bar charts demonstrating dose-response relationship between PRU and both hemorrhage $(\mathbf{A})$ and thrombosis $(\mathbf{B})$ rates. No such relationships were identified using MA-ADP (C and D). 

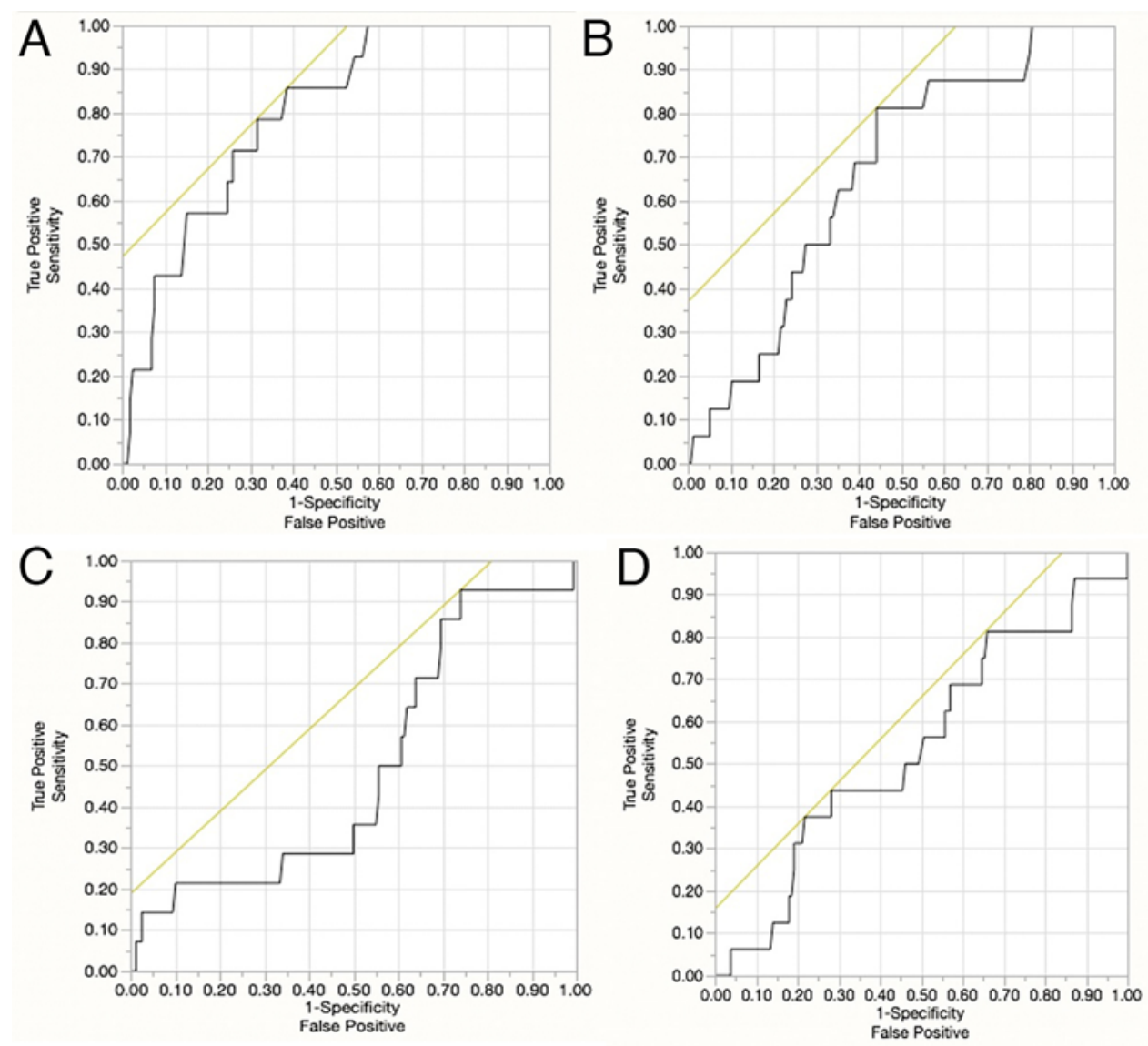

FIG. 4. ROC curves for hemorrhage as predicted by PRU (A), thrombosis as predicted by PRU (B), hemorrhage as predicted by $\operatorname{MA}-A D P(C)$, and thrombosis as predicted by MA-ADP (D). Figure is available in color online only.

third, other nongenetic factors also contribute to clopidogrel hyporesponsiveness, such as medical conditions resulting in malabsorption, use of medications that induce or inhibit hepatic metabolism of the drug (e.g., omeprazole), body habitus, etc. This bewildering assortment of potential pitfalls mandates that focused study be dedicated to this area, and the development of a streamlined protocol to rapidly identify and effectively manage DAPT in endovascular neurosurgery patients would be helpful.

To fulfill that goal, many laboratory tests have been developed to directly assay platelet function in patients on treatment doses of antiplatelet agents. Among neurosurgeons, one of the most widely used of these tests is the VerifyNow P2Y12 PRU assay.,10,14,24 This assay is a whole-blood point-of-care test of P2Y12 receptor activity (the biological target of clopidogrel's antiplatelet action) as measured by turbidimetric optical detection of platelet aggregation. ${ }^{3}$ The mean value in "Plavix response units" for healthy volunteers is 266 PRU with a standard deviation of 42 PRU and a low normal threshold of 182 PRU $(95 \%$ confidence interval up to 197 PRU). The manufacturer's lower threshold of normal is specified as 194 PRU in our hospital's laboratory system.

Despite the conceptual advantages of using preoperative platelet function tests (PFTs), and the benefits demonstrated in the cardiac literature, the same results have proven elusive in endovascular neurosurgery. A metaanalysis ${ }^{24}$ of published results in patients undergoing flow diversion procedures found that only the duration and dose of DAPT contributed to the risk of hemorrhage or stroke. Patients who underwent preoperative PFTs had similar rates of permanent, symptomatic thrombotic complications compared to those who did not (1.5\% vs $1.4 \%)$. While one report ${ }^{9}$ asserted that the ideal range of PRU values in patients undergoing embolization with the Pipeline embolization device (PED) was 70-150 PRU, another study ${ }^{10}$ found no difference in thromboembolic complications among patients with preoperative PRU values in the 200-240 PRU range compared to the 60-200 PRU range. No randomized studies of preoperative PFT use exist for endovascular neurosurgery patients, and none are actively planned. Additionally, no comparative data exist to support the use of one PFT over another, leaving surgeons in a position of uncertainty when employing or interpreting the results of such tests.

Our own published results have raised questions about the accuracy of PRU as a PFT. Using our laboratory's cutoff of 194 PRU to identify clopidogrel responsiveness versus nonresponsiveness, we previously published ${ }^{8}$ a clopidogrel nonresponder rate of $39 \%$ as measured by the PRU, much higher than the upper estimate of $12 \%$ by gold-standard platelet aggregometry in the published literature 21,25 
or $14 \%$ quoted by the FDA. ${ }^{4}$ Given the apparent lack of consistent utility of the PRU demonstrated in the literature and a growing body of evidence in support of a different PFT (TEG-PM) in multiple fields-including trauma/general surgery ${ }_{1}^{16}$ cardiovascular surgery, ${ }^{1,28}$ and endovascular neurosurgery ${ }^{29,33}$ - we began to integrate the TEG-PM into our practice for endovascular neurosurgical patients.

TEG-PM yields more complex data than the PRU. The output of this test is four real-time curves of clot development as a function of time demonstrating the initiation, propagation, and dissolution of a clot. Numerical values extracted from these curves represent multiple aspects of the clotting cascade; the enzymatic initiation of clot formation, strength of the formed clot, and rate of clot breakdown are all part of the basic TEG curve. The PM component includes three curves, the most salient of which represents clot formation in the presence of inhibitors of AA-dependent platelet aggregation. Under these conditions, platelet aggregation and resultant clot strength, represented by the MA value on the TEG, are determined entirely by ADP-dependent platelet aggregation, mediated by the P2Y12 ADP receptor. Our interpretation of the TEG-PM for this study has been primarily focused on the MA-ADP result, as this single numerical value should theoretically measure P2Y12 receptor-mediated platelet aggregation just like the PRU, although the measurement is obtained mechanically by deflection of a fine wire, and not photometrically.

Similar interpretation of the TEG-PM has been successfully utilized at other institutions-primarily outside of the US-for similar indications. One study from China $^{32}$ assigned willing patients undergoing intra- and/ or extracranial internal carotid artery (ICA) stenting with $<50 \%$ inhibition of AA- or ADP-dependent platelet aggregation to triple antiplatelet therapy (TAPT) consisting of $100 \mathrm{mg}$ of aspirin daily, $75 \mathrm{mg}$ of clopidogrel daily, and $100 \mathrm{mg}$ of cilostazol twice daily, rather than their standard DAPT regimen. The TAPT group had a lower rate of major complications within 30 days of stenting, driven by a significant reduction in ischemic complications. Another group from China ${ }^{29}$ observed increased risk of thromboembolic complications in patients undergoing carotid stenting (again, intra- and extracranial) with MA-ADP $>49.95 \mathrm{~mm}$ (AUC 0.65) using similar methodologies to our study and the Daou et al. study. Yet another Chinese study ${ }^{33}$ in patients undergoing Enterprise (Codman \& Shurtleff, Inc.) or Solitaire (Medtronic) stent-assisted coiling of unruptured intracranial aneurysms identified a significantly higher mean MA-ADP in patients with evidence of distal thromboembolism in the vascular territory of the stented vessel on routine postoperative MRI (56.5 vs 46.9 $\mathrm{mm}, \mathrm{p}=0.012$ ). These studies did not compare the results of TEG-PM with PRU.

Our initial observations on the use of TEG-PM were encouraging: correlation with PRU was poor, and the rate of clopidogrel nonresponders was $9 \%$, consistent with the expected number based on previously published results. Unfortunately, implementing the use of TEG-PM was difficult for two key reasons. First, there was no consistency in the literature on which results generated from the testMA-ADP, percentage inhibition of ADP, a different single number, or some combination of values - were best to use. And second, there were no established "safe" values for any of those results. The available literature variously used thresholds of $<50 \mathrm{~mm}$ MA-ADP, ${ }^{29,33}>10 \%$ inhibition of $\mathrm{ADP},{ }^{19}>30 \%$ inhibition of ADP, ${ }^{16}>50 \%$ inhibition of ADP, ${ }^{32}$ and others. Thus, we undertook the tasks of demonstrating the use of TEG in a large, diverse population of endovascular neurosurgical patients, and establishing therapeutic thresholds using similar methodology as had been done by Daou et al. for the PRU in 2016. ${ }^{9}$ We settled on using the MA-ADP as the predictive value because of the similarity to the PRU (discussed above) and because this value is not calculated like the percentage inhibition and reflects absolute platelet function. The percentage inhibition represents platelet function relative to a separate specimen in which both ADP- and AA-mediated platelet aggregation are inhibited. While we have shown that percentage inhibition correlates strongly with MA-ADP, ${ }^{8}$ there does exist a theoretical possibility that strong clots can form in hyperfibrinogenemic patients because of fibrin-mediated platelet aggregation despite profound P2Y12 receptor inhibition, a condition that would not be identified by the percentage inhibition. We have not encountered this situation, nor do we have evidence-based recommendations for its management should it be encountered.

Unfortunately, the present study appears to demonstrate the futility of the TEG-PM MA-ADP in the prediction of complications in endovascular neurosurgery patients. It confirms the utility of the PRU in this regard, is consistent with the results obtained previously by Daou and colleagues, and generalizes their result to a more diverse group of patients. The Daou publication enrolled patients up to July 2014, indicating that likely all patients enrolled underwent placement of a first-generation PED, a device known to harbor a higher risk of complications than the newer Pipeline Flex device ${ }^{6}$ including both distal thromboemboli and the vexing phenomenon of remote intracerebral hemorrhage. It is possible these types of complications are more closely related to degree of platelet inhibition than other complications that may be more directly attributable to technical errors. As a result of this, and the more homogeneous patient population, the results from that study show greater AUC for the ROC curves, as well as a wider optimal PRU range. Still, the AUCs that were observed in the present study ( 0.66 for prediction of thromboembolism, 0.79 for hemorrhage) were comparable to those seen in the publication by Yang and colleagues ${ }^{33}$ ( 0.67 for thromboembolism), the study by Wang et al. ${ }^{29}$ ( 0.654 for thromboembolism), and the study by Daou et al. ${ }^{9}$ (0.7867 for thromboembolism, 0.8412 for hemorrhage).

Our results, while significant, likely suffer in terms of specificity due to the diversity of procedures included and the difficulty in extracting complications that are directly consequent from hyper- or hypo-inhibition of platelet function from those that might result from technical errors. For example, intracerebral hemorrhage resulting from intraoperative aneurysm rupture likely has much more to do with physical trauma to the aneurysm dome by microwires and coils than it does with platelet function, whereas delayed, remote intracerebral hemorrhage after unremarkable placement of a PED is more likely to be 
related to platelet function, perhaps in combination with changes in flow dynamics after the procedure. Similarly, groin hemorrhages may, in part, be related to the initial vessel puncture and decision to place a closure device or execution of the closure device placement. The primary driver of the PRU's success in predicting hemorrhagic complications in this study came from access-site hemorrhages, an event that is becoming rare at our institution as we move toward a radial-first access paradigm. If access-site complications were removed from the analysis, then the PRU mean for neither the hemorrhagic events nor thromboembolic events would have been statistically different from the population means. We cannot say whether the PRU would be predictive of complications were it possible to segregate out those related to technical errors from those related to insufficient or excessive platelet inhibition.

Although we believe that the PRU should be considered the favored test for preoperative PFTs in endovascular neurosurgery patients, these results raise as many questions as they answer. It is not clear why the TEG-PM would fail for this indication. Perhaps more complex analysis of the TEG results is needed to unlock its true predictive power. Alternatively, perhaps it is not an inherent problem with the test, but due to technical difficulty when running the multiple required specimens in the lab. Multicenter trials might reduce this effect. Another explanation might be the diversity of patients enrolled in this study; perhaps threshold values are dependent upon the particular procedure involved. Larger patient samples would be needed to test this hypothesis. Whatever the case, further refinement is needed before the TEG-PM can safely be used to guide decisions about management of antiplatelet agents and whether to proceed with elective endovascular procedures in neurosurgical patients. The role of TEG in general in neurosurgery-with or without PM-remains unproven, but promising reports are proliferating. ${ }^{11,12,17,18,22,27,32}$

\section{Conclusions}

TEG-PM is ineffective in predicting complications in endovascular neurosurgery patients. Its use for this indication is questionable, although further study is necessary in this regard. The PRU is highly sensitive for predicting, in a dose-dependent fashion, both hemorrhagic and thromboembolic complications in this population, although the ideal PRU range is small and technical-rather than purely antiplatelet-mediated-complications may influence this result. When available, we recommend PRU as the preferred PFT for this indication pending randomized headto-head comparisons.

\section{References}

1. Agarwal S, Johnson RI, Shaw M: Preoperative point-of-care platelet function testing in cardiac surgery. J Cardiothorac Vasc Anesth 29:333-341, 2015

2. Brott TG, Hobson RW II, Howard G, Roubin GS, Clark WM, Brooks W, et al: Stenting versus endarterectomy for treatment of carotid-artery stenosis. N Engl J Med 363:11-23, 2010

3. Center for Devices and Radiological Health: 510(k) Substantial Equivalence Determination Decision Summary (Veri-
fyNow ${ }^{\mathrm{TM}}$ PRUTest]. FDA.gov (https://www.accessdata.fda. gov/cdrh_docs/reviews/K141427.pdf) [Accessed December 30, 2019]

4. Center for Drug Evaluation and Research: Reduced Effectiveness of Plavix in Patients Who Are Poor Metabolizers. FDA.gov (www.fda.gov/drugs/postmarket-drug-safety-information-patients-and-providers/fda-drug-safety-communication-reduced-effectiveness-plavix-clopidogrel-patients-whoare-poor) [Accessed December 30, 2019]

5. Chalouhi N, Jabbour P, Gonzalez LF, Dumont AS, Rosenwasser R, Starke RM, et al: Safety and efficacy of endovascular treatment of basilar tip aneurysms by coiling with and without stent assistance: a review of 235 cases. Neurosurgery 71:785-794, 2012

6. Colby GP, Bender MT, Lin LM, Beaty N, Caplan JM, Jiang $\mathrm{B}$, et al: Declining complication rates with flow diversion of anterior circulation aneurysms after introduction of the Pipeline Flex: analysis of a single-institution series of 568 cases. J Neurosurg 129:1475-1481, 2018

7. Colley R, Yan B: Genetic determinations of variable responsiveness to clopidogrel and implications for neurointerventional procedures. Intervent Neurol 1:22-30, 2012

8. Corliss BM, Polifka AJ, Harris NS, Hoh BL, Fox WC: Laboratory assessments of therapeutic platelet inhibition in endovascular neurosurgery: comparing results of the VerifyNow P2Y12 assay to thromboelastography with platelet mapping. J Neurosurg 129:1160-1165, 2018

9. Daou B, Starke RM, Chalouhi N, Barros G, Tjoumakaris S, Rosenwasser RH, et al: P2Y12 reaction units: Effect on hemorrhagic and thromboembolic complications in patients with cerebral aneurysms treated with the Pipeline embolization device. Neurosurgery 78:27-33, 2016

10. Delgado Almandoz JE, Crandall BM, Scholz JM, Fease JL, Anderson RE, Kadkhodayan Y, et al: Pre-procedure P2Y12 reaction units value predicts perioperative thromboembolic and hemorrhagic complications in patients with cerebral aneurysms treated with the Pipeline Embolization Device. J Neurointerv Surg 5 (Suppl 3):iii3-iii10, 2013

11. Ellenberger C, Garofano N, Barcelos G, Diaper J, Pavlovic G, Licker M: Assessment of haemostasis in patients undergoing emergent neurosurgery by rotational elastometry and standard coagulation tests: a prospective observational study. BMC Anesthesiol 17:146, 2017

12. Fulkerson DH, Weyhenmeyer J, Archer JB, Shaikh KA, Walsh M: Thromboelastography-guided therapy of hemorrhagic complications after craniopharyngioma resection: case-based update. Pediatr Neurosurg 54:293-300, 2019

13. Gurbel PA, Bliden KP, Samara W, Yoho JA, Hayes K, Fissha $\mathrm{MZ}$, et al: Clopidogrel effect on platelet reactivity in patients with stent thrombosis: results of the CREST Study. J Am Coll Cardiol 46:1827-1832, 2005

14. Heller RS, Dandamudi V, Lanfranchi M, Malek AM: Effect of antiplatelet therapy on thromboembolism after flow diversion with the pipeline embolization device. J Neurosurg 119:1603-1610, 2013

15. Kakouros N, Kickler TS, Laws KM, Rade JJ: Hematocrit alters VerifyNow P2Y12 assay results independently of intrinsic platelet reactivity and clopidogrel responsiveness. J Thromb Haemost 11:1814-1822, 2013

16. Kasivisvanathan R, Abbassi-Ghadi N, Kumar S, Mackenzie $\mathrm{H}$, Thompson K, James K, et al: Risk of bleeding and adverse outcomes predicted by thromboelastography platelet mapping in patients taking clopidogrel within 7 days of non-cardiac surgery. Br J Surg 101:1383-1390, 2014

17. Kvint S, Schuster J, Kumar MA: Neurosurgical applications of viscoelastic hemostatic assays. Neurosurg Focus 43(5):E9, 2017

18. Lauridsen SV, Hvas CL, Sandgaard E, Gyldenholm T, Mikkelsen R, Obbekjær T, et al: Thromboelastometry shows early 
hypercoagulation in patients with spontaneous subarachnoid hemorrhage. World Neurosurg 130:e140-e149, 2019

19. McTaggart RA, Choudhri OA, Marcellus ML, Brennan T, Steinberg GK, Dodd RL, et al: Use of thromboelastography to tailor dual-antiplatelet therapy in patients undergoing treatment of intracranial aneurysms with the Pipeline embolization device. J Neurointerv Surg 7:425-430, 2015

20. Molyneux AJ, Kerr RS, Yu LM, Clarke M, Sneade M, Yarnold JA, et al: International subarachnoid aneurysm trial (ISAT) of neurosurgical clipping versus endovascular coiling in 2143 patients with ruptured intracranial aneurysms: a randomised comparison of effects on survival, dependency, seizures, rebleeding, subgroups, and aneurysm occlusion. Lancet 366:809-817, 2005

21. Müller I, Besta F, Schulz C, Massberg S, Schönig A, Gawaz M: Prevalence of clopidogrel non-responders among patients with stable angina pectoris scheduled for elective coronary stent placement. Thromb Haemost 89:783-787, 2003

22. Rao A, Lin A, Hilliard C, Fu R, Lennox T, Barbosa R, et al: The utility of thromboelastography for predicting the risk of progression of intracranial hemorrhage in traumatic brain injury patients. Neurosurgery 64 (CN_suppl_1):182-187, 2017

23. Sibbing D, Braun S, Morath T, Mehilli J, Vogt W, Schömig A, et al: Platelet reactivity after clopidogrel treatment assessed with point-of-care analysis and early drug-eluting stent thrombosis. J Am Coll Cardiol 53:849-856, 2009

24. Skukalek SL, Winkler AM, Kang J, Dion JE, Cawley CM, Webb A, et al: Effect of antiplatelet therapy and platelet function testing on hemorrhagic and thrombotic complications in patients with cerebral aneurysms treated with the pipeline embolization device: a review and meta-analysis. J Neurointerv Surg 8:58-65, 2016

25. Slavik L, Ulehlova J, Krcova V, Hlusi A, Indrakova J, Hutyra $\mathrm{M}$, et al: Detection of clopidogrel resistance using ADP induced aggregometry with specific inhibitor PGE1. Clin Lab 60:1475-1480, 2014

26. Spiotta AM, Derdeyn CP, Tateshima S, Mocco J, Crowley RW, Liu KC, et al: Results of the ANSWER trial using the PulseRider for the treatment of broad-necked, bifurcation aneurysms. Neurosurgery 81:56-65, 2017

27. Vahtera AS, Junttila EK, Jalkanen LV, Huhtala HS, Katanandova KV, Hélen PT, et al: Activation of blood coagulation after aneurysmal subarachnoid hemorrhage: a prospective observational trial of rotational thromboelastometry. World Neurosurg 122: e334-e341, 2019

28. Volod O, Lam LD, Lin G, Kam C, Kolyouthapong K, Mac $\mathrm{J}$, et al: Role of thromboelastography platelet mapping and international normalized ratio in defining "normocoagulability" during anticoagulation for mechanical circulatory support devices: a pilot retrospective study. ASAIO J 63:24-31, 2017

29. Wang B, Li XQ, Ma N, Mo D, Gao F, Sun X, et al: Association of thromboelastographic parameters with post-stenting ischemic events. J Neurointerv Surg 9:192-195, 2017
30. Wong JM, Ziewacz JE, Ho AL, Panchmatia JR, Kim AH, Bader AM, et al: Patterns in neurosurgical adverse events: open cerebrovascular neurosurgery. Neurosurg Focus 33(5):E15, 2012

31. Wong JM, Ziewacz JE, Panchmatia JR, Bader AM, Pandey AS, Thompson BG, et al: Patterns in neurosurgical adverse events: endovascular neurosurgery. Neurosurg Focus 33(5):E14, 2012

32. Wu Z, Liu AF, Zhou J, Zhang Y, Wang K, Li C, et al: The safety of triple antiplatelet therapy under thromboelastography guidance in patients undergoing stenting for ischemic cerebrovascular disease. J Neurointerv Surg 11:352-356, 2019

33. Yang H, Li Y, Jiang Y, Lv X: Thromboelastography for monitoring platelet function in unruptured intracranial aneurysm patients undergoing stent placement. Interv Neuroradiol 21:61-68, 2015

\section{Disclosures}

Dr. Hoh reports stock ownership in Propio Vision (a company unrelated to the topic of this paper).

\section{Author Contributions}

Conception and design: Corliss, Harris, Polifka, Fox. Acquisition of data: Corliss, Freedman, Smith, Nerva, Polifka, Hoh, Fox. Analysis and interpretation of data: Corliss, Freedman, Brennan, Fox. Drafting the article: Corliss, Freedman, Brennan. Critically revising the article: Corliss, Brennan, Fox. Reviewed submitted version of manuscript: Corliss, Brennan. Approved the final version of the manuscript on behalf of all authors: Corliss. Statistical analysis: Corliss, Brennan. Administrative/technical/material support: Corliss, Smith, Nerva, Polifka, Hoh, Fox. Study supervision: Smith, Harris, Polifka, Hoh, Fox.

\section{Supplemental Information \\ Online-Only Content}

Supplemental material is available with the online version of the article.

Supplemental Tables 1-3. https://thejns.org/doi/supp1/10.3171/ 2019.12.JNS192396.

\section{Previous Presentations}

Portions of this work were presented at the AANS/CNS Joint Cerebrovascular Section meeting on February 21, 2017, in Houston, Texas, as well as the Florida Neurosurgical Society meeting on August 4, 2019, in Palm Beach, Florida.

\section{Correspondence}

Brian M. Corliss: University of Florida, Gainesville, FL. brianmatthew.corliss@neurosurgery.ufl.edu. 